\section{Making TRAIL}

\section{By Chris Cain, Senior Writer}

Researchers at the Penn State Milton S. Hershey Medical Center have identified a small molecule inducer of TRAIL expression that has antitumor effects in multiple mouse models. ${ }^{1}$ Oncoceutics Inc. has licensed the findings and plans to advance the compound into the clinic this year.

Tumor necrosis factor-related apoptosis-inducing ligand (TRAIL) is an immune cytokine that induces apoptosis by binding tumor necrosis factor receptor superfamily member 10a (TNFRSF10A; DR4; TRAILR1; CD261) and DR5 (TNFRSF10B; TRAILR2; CD262), two receptors expressed on the surface of tumor cells.

Companies have developed recombinant TRAIL derivatives and antibodies targeting DR4 or DR5 to selectively induce apoptosis in tumor cells. However, none of those approaches has advanced into Phase III testing due to poor efficacy. ${ }^{2,3}$

The Penn State researchers took a fresh approach to modulating the TRAIL pathway by screening for small molecule inducers of TRAIL expression. Corresponding author Wafik El-Deiry told SciBX that his team hypothesized that small molecules could be more efficacious than biologics because their superior bioavailability and biodistribution properties would induce high levels of TRAIL in cells throughout the tumor and its surrounding microenvironment.

El-Deiry is professor of medicine and chief of hematology and oncology at the medical center.

The team used a cell-based reporter assay to screen a National Cancer Institute library of about 2,000 compounds for their ability to induce TRAIL expression. The screen identified a small molecule, dubbed TRAIL-inducing compound 10 (TIC10), which led to TRAIL mRNA and protein expression in a panel of cancer cell lines and triggered TRAILdependent cell death at low micromolar concentrations. In mice, the same molecule induced TRAIL expression in tissues including the brain, kidney and spleen.

The next step was to evaluate the efficacy of TIC10 in a series of mouse models for cancer.

In xenograft mice bearing breast or colon cancer cell lines, injection of TIC10 decreased tumor growth compared with vehicle control injection, and its effectiveness was equal or superior to that of recombinant TRAIL. Oral dosing of the compound at $25 \mathrm{mg} / \mathrm{kg}$ also decreased tumor growth compared with vehicle dosing.
In a mouse model for spontaneous lymphoma driven by c-Myc (MYC) overexpression, orally delivered TIC10 prolonged survival.

Finally, the researchers turned to an orthotopic mouse model for glioblastoma multiforme (GBM). Oral delivery of TIC10 decreased tumor growth and doubled overall survival compared with vehicle. When TIC10 was combined with the anti-VEGF antibody Avastin bevacizumab, overall survival tripled.

Roche's Genentech Inc. unit and Chugai Pharmaceutical Co. Ltd. market Avastin to treat several cancers including GBM.

Results were published in Science Translational Medicine.

\section{Pathway finding}

Penn State has exclusively licensed the findings to Oncoceutics, for which El-Deiry is cofounder and CSO. The company was founded in 2009 to develop small molecules with activity against p53-deficient cancers.

TIC10 was effective against both p53-wild-type and p53-mutant cells in vitro and in mouse models. El-Deiry said that the discovery of the compound was a natural extension of his lab's work on understanding tumor-suppressing pathways downstream of p53. "We got into this in the mid-to-late 1990s when we discovered that the TRAIL death receptors were regulated by $\mathrm{p} 53$, and we subsequently showed that TRAIL itself is regulated by p53," he said. "Once we knew that, it became pretty straightforward for us to conceptualize and go after small molecules that could induce TRAIL independent of p53."

Oncoceutics now plans to advance TIC10, which has been renamed ONC201, into a Phase I/II trial in patients with solid tumors, which will include GBM. The trial is being funded by a Pennsylvania Department of Health grant to Oncoceutics. "If it looks safe in the early study, we would like to move into Phase II studies in combination with other targeted cancer therapies in responsive tumor types," El-Deiry said.

He said his lab will continue to work out the precise mechanism by which TIC10 induces TRAIL expression and kills cancer cells.

In the paper, the researchers reported that TIC10 indirectly inhibited protein kinase B (PKB; PKBA; AKT; AKT1) and MEK-MAP kinase (MAPK; ERK) signaling and required the downstream transcription factor forkhead box O3 (FOXO3; FOXO3a) to induce TRAIL expression, but its molecular target or targets were not identified.

"We are absolutely committed to identifying the precise molecular target," El-Deiry said. "We have unpublished evidence that there are some tumor cell lines that respond to TIC10 as a single agent but not to the combination of small molecules that target Akt and ERK, and we plan to study this further to understand why this occurs."

Carl Ware, professor and director of the Infectious and Inflammatory Disease Center at the Sanford-Burnham Medical Research Institute, wanted further confirmation that TIC10's anticancer effects are mediated by TRAIL. 
"The authors provide one experiment utilizing a blocking antibody to TRAIL to demonstrate that the TIC10-induced apoptosis is TRAIL dependent, but the experiment was limited to the original tumor line used to screen for TIC", said Ware. "The use of this or a similar antibody would provide an approach to discriminate between TIC10-induced TRAIL expressed in transplanted human tumors and the bystander effect from TIC10-induced mouse TRAIL. Such experiments would provide more convincing evidence that TRAIL is involved in the action of TIC10 in vivo."

Ware's lab is currently focusing on the role TRAIL plays in the immune system to control viral infection.

Donald Buchsbaum, professor and director of the Division of Radiation Biology at The University of Alabama at Birmingham, wanted to see experiments in TRAIL-resistant cells. "It would have been informative if this paper had reported results for TRAIL-resistant cell lines and their sensitivity to TIC10," he said. "I believe the major limitation to successful clinical translation of TRAIL and agonistic death receptor antibody therapy has been the high incidence of resistance in the majority of patient tumors due to the overexpression of modulators of apoptosis."

The University of Alabama at Birmingham is leading a Phase II trial of Daiichi Sankyo Co. Ltd.'s DR5-agonist antibody tigatuzumab with or without Abraxane nab-paclitaxel in patients with triple-negative breast cancer. Celgene Corp.'s Abraxane is approved to treat non-small cell lung cancer (NSCLC) and metastatic breast cancer.

Aside from tigatuzumab, the fate of programs targeting TRAIL remains unclear.

Human Genome Sciences Inc. had been developing two antibodies before it was acquired by GlaxoSmithKline plc last year-the DR5 agonist lexatumumab and the DR4 agonist mapatumumab. However, GSK had previously obtained rights to the programs under a licensing agreement with HGS that GSK terminated in 2008, calling into question whether the pharma is now interested in pursuing their development. GSK declined to confirm the programs' development status.
Genentech discontinued development of its DR5 agonist antibody RG7425 and recombinant TRAIL in 2009 and currently lists no TRAILor death receptor-targeted drugs in its pipeline. Amgen Inc. declined to confirm the status of its recombinant TRAIL (dulanermin) and does not list any TRAIL programs in its pipeline.

Pennsylvania State University has filed a patent application covering the composition and use of TIC10 to treat cancer, and Oncoceutics exclusively licensed worldwide rights to the compound.

Cain, C. SciBX 6(8); doi:10.1038/scibx.2013.181

Published online Feb. 28, 2013

\section{REFERENCES}

1. Allen, J.E. et al. Sci. Transl. Med.; published online Feb. 6, 2013; doi:10.1126/scitranslmed.3004828

Contact: Wafik S. El-Deiry, Penn State Milton S. Hershey Medical Center, Hershey, Pa.

e-mail: wafik.eldeiry@gmail.com

2. Dimberg, L.Y. et al. Oncogene; published online May 14, 2012; doi:10.1038/onc.2012.164

3. Croft, M. et al. Nat. Rev. Drug Discov. 12, 147-168 (2013)

\section{COMPANIES AND INSTITUTIONS MENTIONED}

Amgen Inc. (NASDAQ:AMGN), Thousand Oaks, Calif. Celgene Corp. (NASDAQ:CELG), Summit, N.J.

Chugai Pharmaceutical Co. Ltd. (Tokyo:4519), Tokyo, Japan

Daiichi Sankyo Co. Ltd. (Tokyo:4568; Osaka:4568), Tokyo, Japan

Genentech Inc., South San Francisco, Calif.

GlaxoSmithKline plc (LSE:GSK; NYSE:GSK), London, U.K.

Human Genome Sciences Inc., Rockville, Md.

National Cancer Institute, Frederick, Md.

Oncoceutics Inc., Hummelstown, Pa.

Penn State Milton S. Hershey Medical Center, Hershey, Pa.

Pennsylvania State University, University Park, Pa.

Roche (SIX:ROG; OTCQX:RHHBY), Basel, Switzerland

Sanford-Burnham Medical Research Institute, La Jolla, Calif. The University of Alabama at Birmingham, Birmingham, Ala. 